Proceedings of the Institute of Mathematics and Mechanics,

National Academy of Sciences of Azerbaijan

Volume 45, Number 2, 2019, Pages 311-318

https://doi.org/10.29228/proc.11

\title{
ON BASICITY OF AN EXPONENTIAL SYSTEM WITH A DISCONTINUOUS PHASE IN THE SOBOLEV SPACE OF PIECEWISE DIFFERENTIABLE FUNCTIONS
}

\author{
VALID F. SALMANOV, VIDADI S. MIRZOYEV, AND SEADET A. NURIYEVA
}

\begin{abstract}
An exponential system with a piecewise linear phase is considered. Necessary and sufficient conditions for basicity of this system in the Sobolev space of piecewise differentiable functions are found.
\end{abstract}

\section{Introduction}

When solving partial differential equations by the Fourier method, there often arise perturbed exponential systems of the following form

$$
\left\{e^{i\left[n t+\gamma_{1}(t)\right]} ; e^{-i\left[k t+\gamma_{2}(t)\right]}\right\}_{n ; k \in N},
$$

where $\gamma_{i}(t), i=1,2$; are in general piecewise continuous functions on the segment $[-\pi, \pi], N$ is the set of all positive integers. The justification of this method requires the study of basis properties of above systems in corresponding function spaces.

Probably the study of basis properties of perturbed systems like (1.1) dates back to the famous works by R. Paley and N. Wiener [14] and N. Levinson [9]. Their basis properties in Lebesgue spaces $L_{p}(-\pi, \pi), p \geq 1$ with $\gamma_{i}(t)=$ $\beta t, i=1,2$, where $\beta$ is a real parameter, have been comprehensively studied in $[10,11,18,19]$, while the case when $\beta$ is a complex parameter has been treated in [8]. In general case, when the functions $\gamma_{i}(t), i=1,2$, are piecewise continuous or simply measurable, basis properties of systems like (1.1) in $L_{p}(-\pi, \pi), p \geq 1$ have been explored in $[1,2,3,4]$.

In case of Sobolev spaces $W_{p}^{1}(-\pi ; \pi)$, the similar problems have been considered in $[12,15]$. Approximation properties of an exponential systems with a discontinuous phase in the space of piecewise continuous functions with the supnorm have been studied in $[7,17,13]$. It should be noted that similar issues have been considered in $[5,16]$.

In this work an exponential system with a piecewise linear phase is considered. Necessary and sufficient conditions for basicity of this system in the Sobolev space of piecewise differentiable functions are found.

2010 Mathematics Subject Classification. 34L10, 46A35.

Key words and phrases. basicity, exponential system Sobolev space. 


\section{Needful information}

Define the Sobolev space of piecewise differentiable functions $K W_{p}^{1}(-\pi ; \pi)$. Denote $I_{1}=(-\pi, 0), I_{2}=(0, \pi)$, and let $I=I_{1} \cup I_{2}$. By $f /{ }_{M}$ we denote the restriction of the function $f$ to the set $M$. Assume

$$
f \in K W_{p}^{1}(-\pi, \pi) \Leftrightarrow f /_{I_{k}} \in W_{p}^{1}\left(I_{k}\right), \quad k=1,2 .
$$

Define the norm in $K W_{p}^{1}(-\pi ; \pi)$ as follows

$$
\|f\|_{K W_{p}^{1}(-\pi, \pi)}=\left(\|f\|_{W_{p}^{1}\left(I_{1}\right)}^{2}+\|f\|_{W_{p}^{1}\left(I_{2}\right)}^{2}\right)^{\frac{1}{2}}
$$

where

$$
\|f\|_{W_{p}^{1}(a, b)}=\|f\|_{p}+\left\|f^{\prime}\right\|_{p}
$$

and $\|\cdot\|_{p}$ is a usual norm in $L_{p}(a, b)$ :

$$
\|f\|_{p}=\left(\int_{a}^{b}|f(t)|^{p} d t\right)^{1 / p}, p \geq 1 .
$$

Denote the direct sum $L_{p}(-\pi, \pi) \oplus C^{2}$ by $L_{p}$, i.e.

$$
L_{p}=L_{p}(-\pi, \pi) \oplus C^{2}
$$

where $C$ is a complex plane. Define the norm in $L_{p}$ as follows:

$$
\|\hat{u}\|_{L_{p}}=\|u\|_{L_{p}}+|\lambda|+|\mu|
$$

where

$$
\hat{u}=(u ; \lambda ; \mu) \in L_{p}, \quad p \geq 1
$$

The following lemma is of vital importance for the main result of this paper.

Lemma 2.1. The operator

$$
A \hat{u}(t)= \begin{cases}\lambda+\int_{-\pi}^{t} u(t) d \tau, & -\pi \leq t<0 \\ \mu+\int_{0}^{t} u(\tau) d \tau, & 0 \leq t \leq \pi\end{cases}
$$

performs an isomorphism between the spaces $L_{p}(-\pi, \pi)$ and $K W_{p}^{1}(-\pi, \pi)$, i.e. the spaces $L_{p}$ and $K W_{p}^{1}$ are isomorphic, $p \geq 1$. 
Proof. The linearity of the operator $A$ is obvious. Let us prove its boundedness. Consider

$$
\begin{array}{r}
\|A \hat{u}\|_{K W_{p}^{1}}^{2}=\|A \hat{u}\|_{W_{p}^{1}(-\pi, 0)}^{2}+\|A \hat{u}\|_{W_{p}^{1}(0, \pi)}^{2}= \\
=\left(\left\|\lambda+\int_{-\pi}^{t} u(\tau) d \tau\right\|_{L_{p}(-\pi, 0)}+\|u\|_{L_{p}(-\pi, 0)}\right)^{2}+ \\
+\left(\left\|\mu+\int_{0}^{t} u(\tau) d \tau\right\|_{L_{p}(0, \pi)}+\|u\|_{L_{p}(0, \pi)}\right)^{2} \leq \\
\leq\left(\|\lambda\|_{L_{p}(-\pi, 0)}+\left\|\int_{-\pi}^{t} u(\tau) d \tau\right\|_{L_{p}(-\pi, 0)}+\|u\|_{L_{p}(-\pi, 0)}\right)^{2}+ \\
+\left(\|\mu\|_{L_{p}(0, \pi)}+\left\|\int_{0}^{t} u(\tau) d \tau\right\|_{L_{p}(0, \pi)}+\|u\|_{L_{p}(0, \pi)}\right)^{2} \leq \\
\leq\left(|\lambda| \pi^{\frac{1}{p}}+\left(\int_{-\pi}^{0}\left(\int_{-\pi}^{0}|u(\tau)| d \tau\right)^{p} d t\right)^{\frac{1}{p}}+\|u\|_{L_{p}(-\pi, 0)}\right)^{2}+ \\
+\left(|\mu| \pi^{\frac{1}{p}}+\left(\int_{0}^{\pi}\left(\int_{0}^{\pi}|u(\tau)| d \tau\right)^{p} d t\right)^{\frac{1}{p}}+\|u\|_{L_{p}(0, \pi)}\right)^{2}= \\
=\left(|\lambda| \pi^{\frac{1}{p}}+\pi^{\frac{1}{p}}\|u\|_{L_{1}(-\pi, 0)}+\|u\|_{L_{p}(-\pi, 0)}\right)^{2}+ \\
+\left(|\mu| \pi^{\frac{1}{p}}+\pi^{\frac{1}{p}}\|u\|_{L_{1}(0, \pi)}+\|u\|_{L_{p}(0, \pi)}\right)^{2}
\end{array}
$$

Applying a simple inequality $\left(a^{2}+b^{2}\right)^{\frac{1}{2}} \leq|a|+|b|$ and then Hölder's inequality, we get

$$
\|A \hat{u}\|_{K W_{p}^{1}(-\pi, \pi)} \leq M_{1}\left(|\lambda|+|\mu|+\|u\|_{L_{p}(-\pi, 0)}+\|u\|_{L_{p}(0, \pi)}\right) .
$$

From the inequality $a^{\frac{1}{p}}+b^{\frac{1}{p}} \leq 2(a+b)^{\frac{1}{p}},(a, b>0)$ it follows

$$
\left(\int_{-\pi}^{0}|u|^{p} d t\right)^{\frac{1}{p}}+\left(\int_{0}^{\pi}|u|^{p} d t\right)^{\frac{1}{p}} \leq 2\left(\int_{-\pi}^{\pi}|u|^{p} d t\right)^{\frac{1}{p}} .
$$

Consequently, we have

$$
\|A \hat{u}\|_{K W_{p}^{1}} \leq M_{2}\left(|\lambda|+|\mu|+\|u\|_{L_{p}(-\pi, \pi)}\right)=M_{2}\|\hat{u}\|_{L_{p}},
$$

i.e. the operator $A$ is continuous.

Now let us show that $\operatorname{Ker} A=\{0\}$. Let $A \hat{u}=0$, i.e.

$$
\left.\begin{array}{l}
\lambda+\int_{-}^{t} \pi u(\tau) d \tau=0, \quad \forall t \in[-\pi, 0), \\
\mu+\int_{0}^{t} u(\tau) d \tau=0, \quad \forall t \in[0, \pi] .
\end{array}\right\}
$$

Differentiating these equalities, we get $u(t)=0$ a.e. on $(-\pi, \pi)$. It follows that $\lambda=\mu=0$. As a result, $\hat{u}=0$. 
Let us show that $\operatorname{Im} A=K W_{p}^{1}(-\pi, \pi)$, where $\operatorname{Im} A$ is the range of the operator $A$. Let $\vartheta \in K W_{p}^{1}(-\pi, \pi)$ be an arbitrary function and $\vartheta_{i}=\vartheta / I_{i}, i=1,2$. Put $\hat{\vartheta}=\left(\vartheta^{\prime} ; \vartheta_{1}(-\pi) ; \vartheta_{2}(0)\right)$. It is clear that $A \hat{\vartheta}=\vartheta$ and $\hat{\vartheta} \in L_{p}$. Then it follows from the Banach inverse operator theorem that the operator $A$ is boundedly invertible. The lemma is proved.

To obtain our main results, we will largely use the following theorems from [2] and [6] for the exponential system

$$
\left\{e^{i[(n+\alpha \operatorname{sign} n) t+\beta \operatorname{sign} n \operatorname{sign} t]}\right\}_{n \in N},
$$

where $\alpha, \beta \in R$ are the real parameters.

Theorem 2.1. [2] Let $-\frac{1}{2 q}<\frac{\beta}{\pi}<\frac{1}{2 p}, p>1, \frac{1}{p}+\frac{1}{q}=1$, and $\omega=2 \alpha+1-\frac{2 \beta}{\pi}$. Then the system (2.1) forms a basis for the space $L_{p}(-\pi, \pi)$ if and only if $-\frac{1}{q}<\omega<\frac{1}{p}$.

Below is the analogue of the classical Hausdorff-Young theorem for the system $(2.1)$.

Theorem 2.2. [6] Let $-\frac{1}{2 q}<\frac{\beta}{\pi}<\frac{1}{2 p}, 1<p \leq 2, \frac{1}{p}+\frac{1}{q}=1$, and $\omega=2 \alpha+$ $1-\frac{2 \beta}{\pi}$. If $\omega>-\frac{1}{q}$, then there exists an absolute constant $M>0$ and the system $\left\{h_{n}^{ \pm}(\cdot)\right\}_{n \in N}$ biorthogonal to (2.1) such that $\forall f \in L_{p}(-\pi, \pi)$ the following inequality holds

$$
\left(\sum_{n=1}^{\infty}\left|a_{n}^{-}\right|^{q}+\sum_{n=1}^{\infty}\left|a_{n}^{+}\right|^{q}\right)^{\frac{1}{q}} \leq M\|f\|_{L_{p}},
$$

where $\left\{a_{n}^{ \pm}(\cdot)\right\}_{n \in N}$ are the biorthogonal coefficients of the function $f(\cdot)$ with respect to the system $\left\{h_{n}^{ \pm}(\cdot)\right\}_{n \in N}: a_{n}^{ \pm}=\int_{-\pi}^{\pi} f(t) \overline{h_{n}^{ \pm}(t)} d t$.

Here

$$
\begin{aligned}
h_{n}^{+}(t)= & \frac{1}{2 \pi}\left(e^{i t}+1\right)_{-1}^{\omega}\left(e^{i t}-1\right)_{+1}^{2 \beta / \pi} e^{-i[(\alpha+1) t+\beta \text { signt }-2 \beta]} \times \\
& \times \sum_{m=0}^{n-1}(-1)^{n-m-1} C_{-2 \beta / \pi}^{n-m-1} \sum_{k=0}^{m} C_{-\omega}^{m-k} e^{-i k t} \\
h_{n}^{-}(t)= & \frac{1}{2 \pi}\left(e^{i t}+1\right)_{-1}^{\omega}\left(e^{i t}-1\right)_{+1}^{2 \beta / \pi} e^{-i[(\alpha+1) t+\beta \text { signt }-2 \beta]} \times \\
& \times \sum_{m=1}^{n}(-1)^{n-m} C_{-2 \beta / \pi}^{n-m} \sum_{k=1}^{m} C_{-\omega}^{m-k} e^{i k t}, n \geq 1,
\end{aligned}
$$

where $(z+1)_{\mp 1}^{\gamma}$ is the branches of the analytic function $(z+1)^{\gamma}$ corresponding in the negative $(-\pi \leq \arg z<\pi)$ and the positive $(0 \leq \arg z<2 \pi)$ directions of the real axes on the complex plane, $C_{\gamma}^{n}=\frac{\gamma(\gamma-1) \ldots(\gamma-n+1)}{n !}$ are binomial coefficients. 


\section{Main result}

Now we state the main result of this work.

Theorem 3.1. Let $-\frac{1}{2 q}<\frac{\beta}{\pi}<\frac{1}{2 p}, \frac{1}{p}+\frac{1}{q}=1, p>1$ and $\omega=2 \alpha+1-\frac{2 \beta}{\pi}$. Then the system

$$
e^{-}(\cdot) \cup e^{+}(\cdot) \cup\left\{e^{i[(n+\alpha \text { signn }) t+\beta \text { signnsignt }]}\right\}_{n \in N}
$$

forms a basis for the space $K W_{p}^{1}(-\pi, \pi)$ if and only if the following inequality holds

$$
-\frac{1}{q}<\omega<\frac{1}{p}
$$

where

$$
e^{-}(t)=\left\{\begin{array}{cc}
1, \quad-\pi \leq t<0, \\
0, \quad 0 \leq t \leq \pi,
\end{array} e^{+}(t)=\left\{\begin{array}{rr}
0, & -\pi \leq t<0 \\
1, & 0 \leq t \leq \pi
\end{array}\right.\right.
$$

and $\alpha_{i}, \beta_{i} \in R, \alpha_{i} \notin\{-N\}, i=1,2$, are the real parameters.

Proof. Let $-\frac{1}{q}<\omega<\frac{1}{p}$. Then, by Theorem 2.1, the system (2.1) forms a basis for the space $L_{p}(-\pi, \pi)$. Let us then prove that the system $\left\{\hat{u}_{n}^{+} ; \hat{u}_{n}^{-}\right\}_{n \in Z_{+}}$ $\left(Z_{+}=\{0\} \cup N\right)$, with

$$
\begin{gathered}
\hat{u}_{0}^{-}=(0 ; 1 ; 0) ; \hat{u}_{0}^{+}=(0 ; 0 ; 1), \\
\hat{u}_{n}^{+}=\left(i\left(n+\alpha_{1}\right) u_{n}^{+}(\cdot) ; u_{n}^{+}(-\pi) ; u_{n}^{+}(0)\right), \\
\hat{u}_{n}^{-}=\left(-i\left(n+\alpha_{2}\right) u_{n}^{-}(\cdot) ; u_{n}^{-}(-\pi) ; u_{n}^{-}(0)\right),
\end{gathered}
$$

where $u_{n}^{+}(t)=e^{i[(n+\alpha) t+\beta \operatorname{sign} t]} ; u_{n}^{-}(t)=e^{-i[(n+\alpha) t+\beta \operatorname{sign} t]}, n \in N$, forms a basis for the space $L_{p}=L_{p} \oplus C^{2}$. It suffices to show that every element $\hat{u}=$ $(u ; \lambda ; \mu) \in L_{p}$ can be uniquely represented in $L_{p}$ in the form of the following series

$$
\hat{u}=\sum_{n=0}^{\infty} c_{n}^{+} \hat{u}_{n}^{+}+\sum_{n=0}^{\infty} c_{n}^{-} \hat{u}_{n}^{-} .
$$

The expansion (3.3) is equivalent to the following one

$$
\begin{gathered}
u(t)=\sum_{n=1}^{\infty} i(n+\alpha) c_{n}^{+} u_{n}^{+}(t)+\sum_{n=1}^{\infty}(-i)(n+\alpha) c_{n}^{-} u_{n}^{-}(t), \\
\mu=c_{0}^{-}+\sum_{n=1}^{\infty} u_{n}^{+}(0) c_{n}^{+}+\sum_{n=1}^{\infty} u_{n}^{-}(0) c_{n}^{-} \\
\lambda=c_{0}^{+}+\sum_{n=1}^{\infty} u_{n}^{+}(-\pi) c_{n}^{+}+\sum_{n=1}^{\infty} u_{n}^{-}(-\pi) c_{n}^{-} .
\end{gathered}
$$

As the system (2.1) forms a basis for the space $L_{p}(-\pi, \pi)$, the expansion of $u(\cdot)$ in the series (3.4) is unique, and the coefficients $\left\{c_{n}^{+} ; c_{n}^{-}\right\}_{n \in N}$ are defined uniquely. 
According to Theorem 2.2, under the conditions (3.2), the Hausdorff-Young type inequality is true for the system (2.1), i.e. the following inequality is true for $1<p \leq 2$ :

$$
\left(\sum_{n=1}^{\infty}\left|(n+\alpha) c_{n}^{+}\right|^{q}+\sum_{n=1}^{\infty}\left|(n+\alpha) c_{n}^{-}\right|^{q}\right)^{1 / q} \leq M\|u\|_{L_{p}}, \frac{1}{p}+\frac{1}{q}=1 .
$$

Applying Hölder's inequality, we get

$$
\begin{gathered}
\sum_{n=1}^{\infty}\left(\left|c_{n}^{+}\right|+\left|c_{n}^{-}\right|\right)=\sum_{n=1}^{\infty}\left(\frac{1}{|n+\alpha|}\left|(n+\alpha) c_{n}^{+}\right|+\frac{1}{|n+\alpha|}\left|(n+\alpha) c_{n}^{-}\right|\right) \leq \\
\leq\left(\sum_{n=1}^{\infty} \frac{1}{|n+\alpha|^{p}}\right)^{1 / p}\left(\sum_{n=1}^{\infty}\left|(n+\alpha) c_{n}^{+}\right|^{q}\right)^{1 / q}+ \\
+\left(\sum_{n=1}^{\infty} \frac{1}{|n+\alpha|^{p}}\right)^{1 / p}\left(\sum_{n=1}^{\infty}\left|(n+\alpha) c_{n}^{-}\right|^{q}\right)^{1 / q}<+\infty
\end{gathered}
$$

It is clear that for $p>2$ the space $L_{p}(-\pi, \pi)$ is continuously embedded into $L_{2}(-\pi, \pi)$. Using Theorem 2.2 again, we have the following inequality

$$
\left(\sum_{n=1}^{\infty}\left|(n+\alpha) c_{n}^{+}\right|^{2}+\sum_{n=1}^{\infty}\left|(n+\alpha) c_{n}^{-}\right|^{2}\right) \leq M\|u\|_{L_{2}} .
$$

Applying the Cauchy-Bunyakovsky inequality, we get

$$
\begin{gathered}
\sum_{n=1}^{\infty}\left(\left|c_{n}^{+}\right|+\left|c_{n}^{-}\right|\right)=\sum_{n=1}^{\infty}\left(\frac{1}{|n+\alpha|}\left|(n+\alpha) c_{n}^{+}\right|+\frac{1}{|n+\alpha|}\left|(n+\alpha) c_{n}^{-}\right|\right) \leq \\
\leq\left(\sum_{n=1}^{\infty} \frac{1}{|n+\alpha|^{2}}\right)^{1 / 2}\left(\sum_{n=1}^{\infty}\left|(n+\alpha) c_{n}^{+}\right|^{2}\right)^{1 / 2}+ \\
+\left(\sum_{n=1}^{\infty} \frac{1}{|n+\alpha|^{2}}\right)^{1 / 2}\left(\sum_{n=1}^{\infty}\left|(n+\alpha) c_{n}^{-}\right|^{2}\right)^{1 / 2}<+\infty .
\end{gathered}
$$

As $\left|u_{n}^{ \pm}(-\pi)\right|=\left|u_{n}^{ \pm}(0)\right|=1$, it is not difficult to see that the coefficients $c_{0}^{ \pm}$in the expansions (3.5) and (3.6) are defined uniquely. So we obtain that $\left\{\hat{u}_{n}^{ \pm}\right\}_{n \in Z_{+}}$ forms a basis for $L_{p}$.

Denote $\vartheta_{n}^{ \pm}=A \hat{u}_{n}^{ \pm}$. As the operator $A$ is an isomorphism between the spaces $L_{p}$ and $K W_{p}^{1}$, it is clear that the system $\left\{\vartheta_{n}^{ \pm}\right\}_{n \in Z_{+}}$forms a basis for $K W_{p}^{1}(-\pi, \pi)$. We have $\vartheta_{0}^{+}(\cdot)=e^{+}(\cdot), \vartheta_{0}^{-}(\cdot)=e^{-}(\cdot)$,

$$
\vartheta_{n}^{+}(t)=e^{i[(n+\alpha) t+\beta \operatorname{sign} t]}, \vartheta_{n}^{-}(t)=e^{-i[(n+\alpha) t+\beta \operatorname{sign} t]}, n \in N .
$$

So we obtain that the system (3.1) forms a basis for the space $K W_{p}^{1}(-\pi, \pi)$.

Let us prove the sufficiency. Let the system (3.1) form a basis for the space $K W_{p}^{1}(-\pi, \pi)$. Then the system $\left\{\hat{u}_{n}^{ \pm}\right\}_{n \in Z_{+}}$also forms a basis for the space $L_{p}$, where

$$
\hat{u}_{0}^{ \pm}=A^{-1}\left(e^{ \pm}(\cdot)\right) ; \hat{u}_{n}^{ \pm}=A^{-1}\left(u_{n}^{ \pm}(\cdot)\right), n \in N
$$




$$
A^{-1}(\vartheta)=\left(\vartheta^{\prime} ; \vartheta_{1}(-\pi) ; \vartheta_{2}(0)\right), \vartheta_{i}=\vartheta /{ }_{I_{i}}, i=1,2 .
$$

We have

$$
\begin{gathered}
\hat{u}_{0}^{+}=(0 ; 0 ; 1), \hat{u}_{0}^{-}=(0 ; 1 ; 0), \\
\hat{u}_{n}^{+}=\left(i(n+\alpha) u_{n}^{+}(\cdot) ; u^{+}(-\pi) ; u^{+}(0)\right), \\
\hat{u}_{n}^{-}=\left(-i(n+\alpha) u_{n}^{-}(\cdot) ; u^{-}(-\pi) ; u^{-}(0)\right), n \in N .
\end{gathered}
$$

Consequently, for every element $\hat{u}=(u ; \lambda ; \mu) \in L_{p}$ we obtain the expansion (3.4)-(3.6), with the coefficients defined uniquely. As a result, we obtain that $\forall u \in L_{p}(-\pi, \pi)$ has a unique expansion of the form (3.4) in $L_{p}(-\pi, \pi)$. In fact, let there exist another expansion for $u(\cdot)$ in $L_{p}$ :

$$
u(t)=\sum_{n=1}^{\infty} i(n+\alpha) b_{n}^{+} u_{n}^{+}(t)+\sum_{n=1}^{\infty}(-i)(n+\alpha) b_{n}^{-} u_{n}^{-}(t) .
$$

Applying Theorem 2.2, it is easy to prove the absolute convergence of the series $\sum\left|b_{n}^{ \pm}\right|$. Let

$$
\begin{gathered}
b_{0}^{-}=\mu-\sum_{n=1}^{\infty} u_{n}^{+}(0) b_{n}^{+}-\sum_{n=1}^{\infty} u_{n}^{-}(0) b_{n}^{-}, \\
b_{0}^{+}=\lambda-\sum_{n=1}^{\infty} u_{n}^{+}(-\pi) b_{n}^{+}-\sum_{n=1}^{\infty} u_{n}^{-}(-\pi) b_{n}^{-} .
\end{gathered}
$$

The basicity of the system $\left\{\hat{u}_{n}^{ \pm}\right\}_{n \in Z_{+}}$for the space $L_{p}$ implies $b_{n}^{ \pm}=c_{n}^{ \pm}, n \in Z_{+}$. Consequently, $u(\cdot)$ can be expanded in a series with respect to the system (3.1) in $L_{p}(-\pi, \pi)$ in a unique way, i.e. the system $(2.1)$ forms a basis for $L_{p}(-\pi, \pi)$. Then, by Theorem 2.1, the condition (3.2) is satisfied. The theorem is proved.

\section{Acknowledgements}

The authors express their deep gratitude to Professor B.T. Bilalov, corresponding member of the National Academy of Sciences of Azerbaijan, for his inspiring guidance and valuable suggestions during the work.

\section{References}

[1] B.T. Bilalov, Basicity of some systems in $L_{p}$, Sb. Primenenie metodov funkts. analiza neklass. urav. mat. fiziki. Novosibirsk. IM SO AN SSSR, (1989) 21-26. (in Russian)

[2] B.T. Bilalov, Basicity of some exponential, sine and cosine systems, Differ. Uravn., 26(1) (1990), 10-16. (in Russian)

[3] B.T. Bilalov, Basis properties of systems of eigenfunctions of some differential operators and their generalizations, Avtoreferat diss. na soisk. uch. st. d.f.-m.n., Moscow, MGU, 25 p. (1995). (in Russian)

[4] B.T. Bilalov, Basis from some exponential system in $L_{p}$, Dokl. RAN., 392(5)(2003), 583-593. (in Russian)

[5] B.T. Bilalov, Ch.M. Hashimov, On Decomposition In Banach Spaces Proceedings of the Institute of Mathematics and Mechanics, National Academy of Sciences of Azerbaijan, 40(2) (2014), 97-106.

[6] B.T. Bilalov, M.Kh. Karakash, The Hausdorff-Young type theorem for one system of exponents, Trans. Sci. Azer., XXIV(4) (2004), 19-23. (in Russian) 
[7] B.T. Bilalov, V.F. Salmanov, Uniform convergence of biorthogonal series on one system of exponents, Transactions of NAS of Azer., XXIII(4) (2003), 25-34.

[8] G.G. Devdariani, On basicity of a system of functions, Differ. Uravn., 22(1) (1986), 170-171. (in Russian)

[9] N. Levinson, Gap and density theorems, New York, Publ, Amer. Math.-Soc., 123128, (1940).

[10] E.I. Moiseev, On basicity of sine and cosine systems, DAN SSSR 275(4) (1984), 794-798. (in Russian)

[11] E.I. Moiseev, On basicity of a sine system, Differ. Uravn., 23(1) (1987), 177-179. (in Russian)

[12] E.I. Moiseev, On basicity of sine and cosine systems in weighted space, Differ. Uravn., 34(1) (1998), 40-44. (in Russian)

[13] E.I. Moiseev, N.O. Taranov, Solution of a Gellerstedt problem for LavrentievBitsadze equation, Differ. Uravn., 45(4) (2009), 543-548. (in Russian)

[14] R. Paley, N. Wiener, Fourier transform in complex domain, Moscow, Nauka (1964) (in Russian)

[15] D.L. Russell, On exponential bases for the Sobolev spaces over an interval, Journal of Mathem. Anal. and Appl., 87 (1982), 528-550.

[16] S.R. Sadigova, Z.A. Kasumov, On atomic decomposition for Hardy classes with respect to degenerate exponential systems, Proceedings of the Institute of Mathematics and Mechanics, National Academy of Sciences of Azerbaijan, 40(1) (2014), $55-67$.

[17] V.F. Salmanov, Completeness and minimality of exponential system in the space of piecewise continuous functions, The scientific and pedagogical news of Odlar Yurdu University, Baku (2005), 42-45. (in Russian)

[18] A.M. Sedletski, Biorthogonal expansions of functions in series of exponents on intervals of the real axis, Russian Math. Surveys, 37(5) (1982), 57108.

[19] A.M. Sedletski, On convergence of non-harmonic Fourier series in exponential, sine and cosine systems, DAN SSSR, 301(5), 501-504 (1988), 170-171. (in Russian)

Valid F. Salmanov

Azerbaijan State Oil and Industry University, Baku, Azerbaijan.

E-mail address: valid.salmanov@mail.ru

Vidadi S. Mirzoyev

Institute of Mathematics and Mechanics of NAS of Azerbaijan, Baku, Azerbaijan.

Seadet A. Nuriyeva

Azerbaijan Tourism and Management University

E-mail address: sada.nuriyeva@inbox.ru

Received: June 13, 2019; Revised: October 3, 2019; Accepted: October 7, 2019 